\title{
Gene-Environment Interactions With Cognition in Late Life and Compression of Morbidity
}

$\mathrm{R}$

apid research advances in Alzheimer's disease over the past three decades have put us in a position to offer therapy delaying disease progression, thus delaying nursing home admission, and there is the promise of true disease-modifying agents in the near future. There is an important genetic risk for Alzheimer's disease associated with the $\mathrm{E}^{*} 4$ allele of the apolipoprotein gene (APOE*4) (1). With our increasing understanding of the clinical and pathophysiological characteristics of Alzheimer's disease, attention is now focusing on the earliest manifestations of the disease in an effort to identify people at risk who might benefit from new therapies-including individuals who are cognitively normal. At the same time, new advances are being made in the nonpharmacological treatment of Alzheimer's disease and related dementias in recognition of the current limitations in more traditional treatment regimens.

Alzheimer's disease is now seen as a disease with multiple risk factors. Although many of them may be causal, the majority are thought to be risk modifiers in that they can accelerate or decelerate the time to develop the clinical manifestations of the disease, i.e., the dementia itself. Although there is nothing that we can do about our genetic makeup, altering other factors may play an important role in the natural history of the disease. Two articles in this issue of the Journal make these points explicitly.

"Until such time as

treatments are available

that can truly modify or

prevent Alzheimer's

disease, improving and

extending the period of

higher quality of life may

be the most important

clinical outcome."

Chen and colleagues analyzed the rate of change of whole brain volumes as a function of APOE*4 "load" in cognitively normal subjects. They found that individuals who had two copies of the $E^{*} 4$ allele had more rapid loss of brain tissue than individuals either with a single copy or who were $\mathrm{E}^{*} 4$ negative. This collaborative study used two different techniques-brain boundary shift integration and iterative principal components analysis. The analyses were completed at two different laboratories using the same data set of anatomical magnetic resonance imaging (MRI) scans from 36 cognitively normal subjects; each of these individuals was scanned twice approximately 2 years apart. Brain boundary shift integration uses the movement ("shift") of the brain-CSF boundary as the measure of diffuse atrophy. The change in volume over time is standardized into a rate of change relative to each subject's own baseline MRI scan. The iterative principal components analysis, by contrast, establishes which portions of the brain had a significantly greater change in intensity than the typical intensity change within the whole brain. The total volume of the brain that is determined to have had significantly greater change is then quantified as volume gain (or relocation) or volume loss (atrophy) as a rate of change over time.

Both techniques found statistically large and significant associations between $\mathrm{APOE}^{*} 4$ load and the annual rate of whole brain atrophy. Although both techniques demonstrated a significant difference between the atrophy rates for the $\mathrm{E}^{*} 4$ homozygotes and those of the noncarriers, the statistically smaller effects of the heterozygotes versus the homozygotes were not significant (and would have required 50-80 total cases to be adequately powered). 
These findings are even more striking because almost without exception, these individuals remained cognitively normal throughout the follow-up period and beyond. Further follow-up of this important group of at-risk individuals will allow Chen and colleagues the opportunity to describe both the whole brain changes as individuals transition to Alzheimer's disease and also the regionally specific changes that may serve as sensitive and specific biomarkers of the trajectory toward Alzheimer's disease.

As much as genotype plays an important and still immutable role in the natural history of Alzheimer's disease, Wilson and colleagues have found evidence that environmental factors can affect the way in which individuals with moderate to severe Alzheimer's disease adapt to their environment. Specifically, the transition to nursing home care is made easier if the patient has had prior experience in a day care setting. They examined the cognitive function of more than 400 Alzheimer's disease patients over a 4year period as the patients made the transition from home to institutional care. Placement in a nursing home resulted in a decrease in performance on measures of cognition-due in part to the progression of the disease but also, more than likely, to the disruptive effects of the move on a range of adaptive functions. However, if the patient had been in a day care setting before admission to the nursing home, then the accelerated cognitive decline while in the nursing home was essentially eliminated. The effect was powerful enough to attenuate the association between higher educational attainment and more rapid decline after nursing home admission.

The authors discussed their findings in the context of the potentially beneficial effects of nonspecific experience in an institutional setting (i.e., day care) on the adaptation to the nursing home environment. It may also be the case, as noted by the authors, that individuals who use day care services-or the families of those patients-differ in important ways from those who do not.

However, it is also possible that the day care setting itself may have had a beneficial effect on cognition and adaptive functions. The day care environment, even those with limited structured programs, may provide increased social contacts, opportunities for cognitive stimulation, and physical exercise. Individually these different activities may have limited efficacy, but in combination they may have beneficial effects.

Cognitive stimulation in the context of a day care setting does have a beneficial effect on mental status (2). Alzheimer's disease patients participating in a psychostimulation program integrated into a day care center had a mean increase in scores on the MiniMental State Examination of 1.13 points over a 24 -week observation period (versus a 1.5-point decline in the control group). Although the dementia of the patients in that study was much less severe than that of the patients evaluated by Wilson and colleagues, the main point is the same: maintaining as much contact as possible with the outside world and exercising the mind and body likely have positive and beneficial effects on the natural history of Alzheimer's disease up to and including the time after nursing home placement.

How might these changes occur? Before the emergence of medications to treat the symptoms of Alzheimer's disease, cognitive stimulation was thought to be potentially beneficial for dementia patients. Although cognitive stimulation does not always appear efficacious and methodological problems may limit the validity of some studies (3), certain cognitive domains may benefit from these interventions (4). All of these findings are in line with the emerging line of studies that demonstrate that Alzheimer's disease patients retain a remarkable degree of CNS plasticity and adaptability. Although many of these mechanisms are observed early in the course of the disease (e.g., references 5 and 6), pathological studies conducted in biopsied Alzheimer's disease patients with mild/moderate dementia have shown increased synaptic contact size (7).

To date, there is no evidence that therapies targeted at Alzheimer's disease alter the time to death (e.g., reference 8), although time to cognitive (9) and noncognitive outcomes (8) are extended. To the extent that factors such as APOE* 4 status affect the un- 
derlying neuropathology, we can imagine that multiple factors expand or compress dementia-free time or time with higher quality of life (see Figure 1). This idea is related directly to the concept of compression of morbidity (10), which posits the ideal of a long(er), higher quality life with a relatively short(er) period of terminal decline. In the present context, illness burden (i.e., the presence of clinical dementia) is reduced by both pharmacological and nonpharmacological means with an end result of a "compression" of the time of most severe dementia until death.

FIGURE 1. Effects of Modification of the Natural History of Alzheimer's Disease ${ }^{a}$

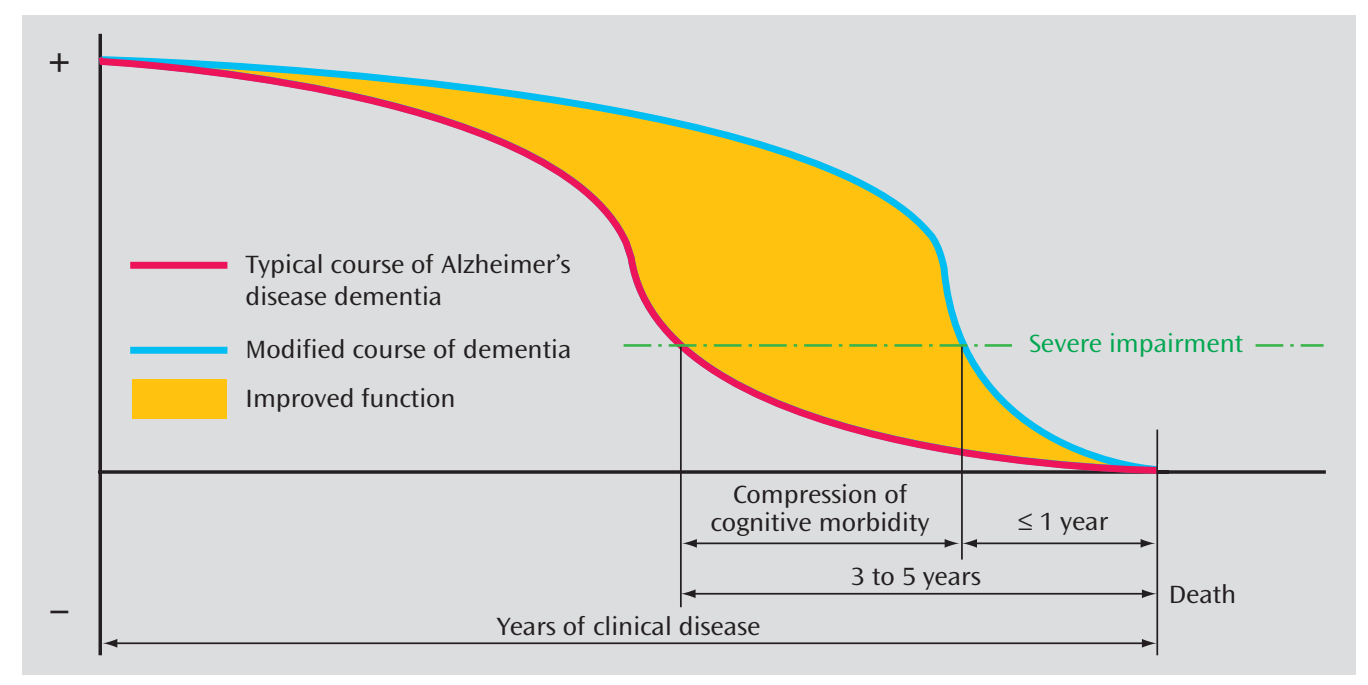

${ }^{a}$ Genetic factors, such as apolipoprotein gene load, affect the earliest stages of the disease, while symptomatic treatment, both pharmacological and nonpharmacological, expands the period of higher quality of life and compresses the period of cognitive morbidity -in this case from 3-5 years to less than 1 year. Image courtesy of Sr. Tarraga.

Environment interactions and their effects on the natural history of Alzheimer's disease are important in developing rational treatment management strategies. We cannot alter our genes (yet), and these confer an additional burden on the CNS and result in earlier expression of the dementia. However, we can alter our environment, and this appears to expand the time of higher quality of life (defined as better mental status or time outside of nursing home care). Until such time as treatments are available that can truly modify or prevent Alzheimer's disease, improving and extending the period of higher quality of life may be the most important clinical outcome. These two studies reported in the Journal demonstrate the importance of understanding these complex genetic and environmental factors in modifying the natural history of Alzheimer's disease.

\section{References}

1. Saunders AM, Hulette C, Welsh-Bohmer KA, Schmechel DE, Crain B, Burke JR, Alberts MJ, Strittmatter WJ, Breitner JC, Rosenberg C: Specificity, sensitivity, and predictive value of apolipoprotein-E genotyping for sporadic Alzheimer's disease. Lancet 1996; 348:90-93

2. Tarraga L, Boada M, Modinos G, Espinosa A, Diego S, Morera A, Guitart M, Balcells J, Lopez OL, Becker JT: A randomised pilot study to assess the efficacy of an interactive, multimedia tool of cognitive stimulation in Alzheimer's disease. J Neurol Neurosurg Psychiatry 2006; 77:1116-1121

3. Grandmaison E, Simard M: A critical review of memory stimulation programs in Alzheimer's disease. J Neuropsychiatry Clin Neurosci 2003; 15:130-144

4. Clare L, Woods RT, Moniz-Cook ED, Orrell M, Spector A: Cognitive rehabilitation and cognitive training for early-stage Alzheimer's disease and vascular dementia (review). Cochrane Database Syst Rev $2003 ; 4$ : CD003260

5. Kemperman G, Gast D, Gage FH: Neuroplasticity in old age: sustained fivefold induction of hippocampal neurogenesis by long-term environmental enrichment. Ann Neurol 2002; 52:135-143 
6. Herbster AN, Nichols T, Wiseman MB, Mintun MA, DeKosky ST, Becker JT: Functional connectivity in auditory verbal short-term memory in Alzheimer's disease. Neuroimage 1996; 4:67-77

7. DeKosky ST, Scheff SW: Synapse loss in frontal cortex biopsies in Alzheimer's disease: correlations with cognitive severity. Ann Neurol 1990; 27:457-464

8. Lopez OL, Becker JT, Wisniewski S, Saxton J, Kaufer DI, DeKosky ST: Cholinesterase inhibitor treatment alters the natural history of Alzheimer's disease (abstract). J Neurol Neurosurg Psychiatry 2002; 72:310

9. Lopez OL, Becker JT, Saxton J, Sweet R, Klunk W, DeKosky ST: Alteration of a clinically meaningful outcome in the natural history of Alzheimer's disease by cholinesterase inhibition. J Am Geriatr Soc 2005; 53:83-87

10. Fries JF: Aging, natural death, and the compression of morbidity. N Engl J Med 1980; 303:130-135

JAMES T. BECKER, PH.D. LUIS TARRAGA MESTRE, M.SC. SCOTT ZIOLKO, B.S. OSCAR L. LOPEZ, M.D.

Address correspondence and reprint requests to Dr. Becker, Neuropsychology Research Program, Suite 830, 3501 Forbes Ave., Pittsburgh, PA 15213; beckerjt@msx.upmc.edu (e-mail).

Dr. Becker has been a consultant for Grifols S.A. and received an advisory panel payment from Forest. Dr. Lopez has been a consultant for and on the advisory panel for Eisai/Pfizer and Forest and a consultant for Grifols and Servier. The remaining authors report no competing interests. 